\title{
CONTROLE QUÍMICO DA ANTRACNOSE (Colletotrichum graminicola) DO SORGO
}

\author{
NICÉSIO FILADELFO JANSSEN DE ALMEIDA PINTO ${ }^{1}$ \\ ${ }^{1}$ Pesquisador, Embrapa Milho e Sorgo. Caixa Postal 151, CEP. 35701-970 Sete Lagoas, MG. E- mail: \\ nicesio@cnpms.embrapa.br (autor para correspondência).
}

Revista Brasileira de Milho e Sorgo, v.2, n.3, p.148-152, 2003

\begin{abstract}
RESUMO - O objetivo deste trabalho foi avaliar a eficiência de fungicidas no controle da antracnose foliar do sorgo e seus reflexos na qualidade sanitária e peso de sementes. Os seguintes fungicidas ( $\mathrm{g}$ i. a. ha ${ }^{-1}$ ) foram aplicados na variedade de sorgo BR 009B: tebuconazole (200), benomyl (500), mancozeb (2400), triforine (285), fenarimol (120), prochloraz (450), imibenconazole (150), azoxystrobin (150), chlorothalonil (1125), captan (750) e carbendazim (350). Parcelas sem tratamento com fungicida constituíram a testemunha. Utilizou-se o delineamento experimental de blocos ao acaso, com 12 tratamentos em três repetições. Cada parcela foi constituída por quatro fileiras de $7 \mathrm{~m}$ de comprimento e distava $2 \mathrm{~m}$ das demais. As aplicações dos fungicidas, utilizando-se pulverizador costal manual, foram em número de três e realizadas em intervalos de dez dias, iniciando-se quando as plantas apresentavam incidência moderada da antracnose foliar. As avaliações da severidade da antracnose foram realizadas aos zero, 20 e 45 dias após a primeira aplicação, por meio de escala de notas de 0 a $5(0=$ ausência de lesões foliares e $5=$ lesões em $100 \%$ das folhas, com seca das plantas). As sementes foram submetidas à análise de sanidade pelo método do papel de filtro com congelamento e, para a quantificação da produção, os pesos das sementes foram ajustados para 13\% de umidade. Os resultados obtidos mostraram que os fungicidas prochloraz, carbendazim, benomyl e azoxystrobin foram os mais eficientes no controle da antracnose foliar, tendo-se como parâmetro o progresso da doença entre as avaliações inicial e a realizada aos 45 dias após o início das pulverizações. Os resultados também evidenciaram que a menor incidência de C. graminicola nas sementes ocorreu nos tratamentos com carbendazim, azoxystrobin e prochloraz; e que os maiores pesos de sementes foram obtidos com os tratamentos prochloraz, carbendazim, benomyl e azoxystrobin.
\end{abstract}

Palavras-chave: Sorghum bicolor, fungicidas, sanidade de sementes.

\section{CHEMICAL CONTROL OF THE ANTHRACNOSE (Colletotrichum graminicola) OF SORGHUM}

\begin{abstract}
The following fungicides ( $\mathrm{g}$ a. i. ha ${ }^{-1}$ ) were applied on the sorghum variety BR 009B: tebuconazole (200), benomyl (500), mancozeb (2400), triforine (285), fenarimol (120), prochloraz (450), imibenconazole (150), azoxystrobin (150), chlorothalonil (1125), captan (750) and carbendazim (350). Plots without fungicide treatment constituted the control. Treatments were laid out in a randomized complete block design ( 12 treatments in 3 replications) with plots of four rows, $7 \mathrm{~m}$ long and $2 \mathrm{~m}$ apart. Applications, in number of three, were performed every 10 days with a costal sprayer. Fungicides were first applied when plants presented a moderate incidence of leaf anthracnose. Anthracnose evaluations were made at the day of the first application and 20 and 45 days later, using a 0 to 5 scale $(0=$ no symptoms and $5=$ dead
\end{abstract}


plants). Seeds were submitted to a seed health test through the deep freezing method, and, for the quantification of the production, the weights of the seeds were adjusted for $13 \%$ of humidity. Results showed that the fungicides prochloraz, carbendazim, benomyl and azoxystrobin were the most efficient in the control of leaf anthracnose, as indicated by the anthracnose progress within the 45 days interval of evaluation. The results also evidenced that the smallest incidence of $C$. graminicola on seeds happened in the treatments with carbendazim, azoxystrobin and prochloraz; and that the largest weights of seeds were obtained with the treatments prochloraz, carbendazim, benomyl and azoxystrobin.

Key words: Sorghum bicolor, fungicides, seed health.

No Brasil, a antracnose causada pelo fungo Colletotrichum graminicola (Ces.) Wils. é uma das mais importantes doenças da cultura do sorgo, ocorrendo em todas as regiões de plantio desse cereal. Sob condições ambientais, favoráveis pode causar, em cultivares suscetíveis, reduções consideráveis de produção e de qualidade das sementes ou grãos (Casela et al., 1992). Essas regiões incluem os trópicos semi-árido e úmido e regiões de clima temperado, com temperaturas elevadas no verão (Tarr, 1962). Nas áreas onde a antracnose ocorre com maior severidade, a mesma torna-se um fator limitante para o desenvolvimento da cultura do sorgo (Harris et al., 1964). A antracnose foliar pode limitar a produção em muitas regiões, com redução na produção de grãos em 50\% ou mais, em epidemias severas (Warren, 1986). O fator mais comum entre todas as regiões de ocorrência da antracnose é a presença de chuvas freqüentes, principalmente durante o estádio de crescimento da cultura do sorgo. A fase foliar da doença pode ocorrer em qualquer estádio de desenvolvimento da planta, mas aparece normalmente a partir do início de desenvolvimento da panícula (Casela et al., 1992).

Embora o uso de cultivares resistentes se constitua em estratégia eficiente e econômica de controle da antracnose, tal medida é dificultada pela alta variabilidade apresentada por C. graminicola, a qual determina, muitas vezes, a rápida adaptação do patógeno às cultivares resistentes (Casela \& Ferreira, 1991). Outra medida de controle que pode ser utilizada é a mistura de cultivares apresentando reações de suscetibilidade, resistência e resistência intermediária (Guimarães et al., 1998). A avaliação da resistência de genótipos de sorgo à antracnose também tem sido motivo de estudo (Guimarães et al., 1999). Uma estratégia emergencial que vem sendo utilizada em campos de linhagens e de produção de sementes de sorgo visando o controle da antracnose tem sido a aplicação de fungicidas. Entretanto, há poucas informações sobre a eficiência desta tecnologia no controle de C. graminicola.

Segundo Pinto (1998), no controle da antracnose foliar do sorgo (C. graminicola) destacou-se o fungicida prochloraz. Adicionalmente, Pinto (1999) relata que os fungicidas prochloraz, tebuconazole e a mistura propiconazole + difenoconazole foram eficientes no controle de $C$. graminicola, proporcionando aumento no peso das sementes de sorgo da cultivar BR 304.

Entre os tratamentos testados por Valarini et al. (1988) para o controle de C. graminicola, os fungicidas mais eficientes foram carbendazim + thiram, captafol, iprodione + thiram, captafol + pentacloronitrobenzeno, captan, guazatine + imazalil, benomyl e thiram.

O objetivo deste trabalho foi avaliar a eficiência de fungicidas no controle da antracnose foliar do sorgo e seus reflexos na qualidade sanitária e no peso das sementes.

O presente trabalho foi conduzido no município de Sete Lagoas, MG, na área experimental da Embrapa Milho e Sorgo, sendo o plantio realizado 
em fevereiro de 1999. Os seguintes fungicidas ( $\mathrm{g}$ i. a. $\left.\mathrm{ha}^{-1}\right)$ foram aplicados na variedade de sorgo BR 009B (altamente suscetível à antracnose): tebuconazole (200), benomyl (500), mancozeb (2400), triforine (285), fenarimol (120), prochloraz (450), imibenconazole (150), azoxystrobin (150), chlorothalonil (1125), captan (750) e carbendazim (350). Parcelas sem tratamento com fungicida constituíram a testemunha.

Utilizou-se o delineamento experimental de blocos ao acaso, com 12 tratamentos em três repetições. Cada parcela foi constituída por quatro fileiras de $7 \mathrm{~m}$ (média 12 plantas $/ \mathrm{m}$ ) e distanciada $2 \mathrm{~m}$ das demais. Foram realizadas três aplicações dos fungicidas com pulverizador costal manual, em intervalos de dez dias, iniciando-se aos 45 dias após o plantio, quando as plantas de todas as parcelas apresentavam incidência moderada da antracnose foliar.

Nas avaliações da eficiência dos tratamentos, realizadas nas duas fileiras centrais das parcelas, aos zero, 20 e 45 dias após a primeira pulverização fungicida, foi utilizada uma escala de notas proposta por Pinto \& Fernandes (1995): $0=$ ausência de lesões foliares, $1=$ lesões esparsas, $2=$ lesões em $50 \%$ das folhas, com $25 \%$ de severidade, $3=$ lesões em $75 \%$ das folhas, com $50 \%$ de severidade, $4=$ lesões em $100 \%$ das folhas, com $75 \%$ de severidade e $5=$ lesões em $100 \%$ das folhas, com seca das plantas.

Avaliou-se a incidência de C.graminicola nas sementes de sorgo pelo método do papel de filtro com congelamento (Limonard, 1966), através da análise da sanidade de 400 sementes por parcela (quatro repetições de 100 sementes). Para a determinação do peso das sementes das duas fileiras centrais, com base nas umidades das sementes de todas as parcelas experimentais, estas foram ajustadas para $13 \%$ de umidade. Para a determinação dos teores de umidade das sementes, utilizou-se o método de estufa com circulação natural de ar, regulada em 105
$+1{ }^{\circ} \mathrm{C}$, durante 24 horas, de acordo com as Regras de Análise de Sementes (Brasil, 1992).

Os resultados contidos na Tabela 1 mostram que os fungicidas benomyl, prochloraz, azoxystrobin e carbendazim foram os mais eficientes no controle da antracnose foliar, notas médias $<2,0$ (lesões em $50 \%$ das folhas, com $25 \%$ de severidade), tendo-se como parâmetro o progresso da doença entre a avaliação inicial (zero dia) e a realizada aos 45 dias após o início das pulverizações com fungicidas. A eficiência obtida neste trabalho com o fungicida prochloraz no controle de $C$. graminicola nas folhas do sorgo está em conformidade com o relatado por Pinto (1998). Com referência às eficiências apresentadas pelos fungicidas benomyl, azoxystrobin e carbendazim, nenhuma referência foi encontrada na literatura consutada.

Os resultados contidos na Tabela 2 evidenciam que a menor incidência de $C$.graminicola nas sementes de sorgo ocorreu nos tratamentos com carbendazim, azoxystrobin, prochloraz e benomyl. Resultados semelhantes com o fungicida benomyl no controle desse fungo em sementes de sorgo foram obtidos por Valarini et al. (1988), os quais também mostram a eficiência da mistura carbendazim + thiram no controle desse fungo.

Os maiores pesos de sementes, oriundas da colheita das panículas das duas fileiras centrais das parcelas, foram obtidos com os tratamentos prochloraz (incremento de produção em 113,7 \%), carbendazim, benomyl e azoxystrobin. Resultados semelhantes em tratamento profilático de panículas de sorgo com os fungicidas propiconazole, propiconazole + difenoconazole e tebuconazole foram obtidos por Pinto (1999).

Do exposto, concluiu-se que os fungicidas prochloraz, carbendazim, benomyl e azoxystrobin apresentam alta eficiência no controle da antracnose foliar do sorgo, na redução da incidência de $C$. graminicola nas sementes e no aumento do peso das sementes de sorgo. 
TABELA 1. Controle químico da antracnose foliar (Colletotrichum graminicola) em sorgo, cultivar BR 009B, aos 20 e 45 dias após a primeira aplicação de fungicidas. Embrapa Milho e Sorgo, Sete Lagoas, MG, 1999.

\begin{tabular}{lcccc}
\hline Tratamento & Dose $\left(\mathbf{g}\right.$ i.a. $\left.\mathbf{h a}^{\mathbf{- 1}}\right)$ & Zero dia & $\mathbf{2 0}$ dias & 45 dias \\
\hline Tebuconazole & 200,0 & $1,5 *$ & 1,8 & 3,0 \\
Benomyl & 500,0 & 1,2 & 1,5 & 1,7 \\
Mancozeb & 2400,0 & 2,0 & 2,5 & 2,8 \\
Triforine & 285,0 & 1,5 & 1,5 & 2,2 \\
Fenarimol & 120,0 & 1,7 & 2,3 & 3,5 \\
Prochloraz & 450,0 & 1,0 & 1,0 & 1,3 \\
Imibenconazole & 150,0 & 1,2 & 1,8 & 3,0 \\
Azoxystrobin & 150,0 & 1,0 & 1,2 & 1,5 \\
Chlorothalonil & 1125,0 & 1,3 & 2,3 & 2,3 \\
Captan & 750,0 & 1,3 & 2,3 & 3,3 \\
Carbendazim & 350,0 & 1,0 & 1,0 & 1,3 \\
Testemunha & ----- & 1,7 & 2,5 & 4,5 \\
\hline
\end{tabular}

* Escala de notas variando de 0 a 5 (Pinto \& Fernandes, 1995).

TABELA 2. Incidência de Colletotrichum graminicola em sementes de sorgo da cultivar BR 009B e produção de sementes em plantas pulverizadas com fungicidas. Embrapa Milho e Sorgo, Sete Lagoas, MG, 1999.

\begin{tabular}{lccc}
\hline Tratamento & Dose $\left(\mathbf{g}\right.$ i.a. $\left.\mathbf{~ h a}^{-\mathbf{1}}\right)$ & C. graminicola $\mathbf{( \% )}$ & Produção $\left(\mathbf{k g ~ h a} \mathbf{~}^{\mathbf{1}}\right)^{*}$ \\
\hline Prochloraz & 450,0 & $0,7 \mathrm{gh}$ & $7542,0 \mathrm{a}$ \\
Carbendazim & 350,0 & $0,2 \mathrm{~h}$ & $7218,0 \mathrm{a}$ \\
Benomyl & 500,0 & $4,0 \mathrm{fg}$ & $6588,0 \mathrm{ab}$ \\
Azoxystrobin & 150,0 & $0,3 \mathrm{~h}$ & $6534,0 \mathrm{ab}$ \\
Tebuconazole & 200,0 & $9,0 \mathrm{~d}$ & $4554,0 \mathrm{bc}$ \\
Triforine & 285,0 & $5,0 \mathrm{ef}$ & $4338,0 \mathrm{bc}$ \\
Chlorothalonil & 1125,0 & $8,3 \mathrm{de}$ & $4122,0 \mathrm{c}$ \\
Imibenconazole & 150,0 & $16,0 \mathrm{ab}$ & $4104,0 \mathrm{c}$ \\
Fenarimol & 120,0 & $10,3 \mathrm{~cd}$ & $3816,0 \mathrm{c}$ \\
Mancozeb & 2400,0 & $8,0 \mathrm{de}$ & $3798,0 \mathrm{c}$ \\
Captan & 750,0 & $13,0 \mathrm{bc}$ & $3762,0 \mathrm{c}$ \\
Testemunha & ---- & $18,0 \mathrm{a}$ & $3528,0 \mathrm{c}$ \\
\hline C.V. (\%) & \multicolumn{3}{c}{15,0} \\
\hline
\end{tabular}

* Peso ajustado a $13 \%$ de umidade, em base úmida.

\section{Agradecimentos}

A Osni Alves da Silva, Ademar Verneque e José Moreira Campos (Assistentes de Pesquisa),
Gilberto Ribeiro Rodrigues e Almir Roberto da Silva (Operários Rurais), a valiosa contribuição na condução deste trabalho. 


\section{Literatura Citada}

BRASIL. Ministério da Agricultura e Reforma Agrária. Regras para Análise de Sementes. Brasília, 1992. 365p.

CASELA, C.R.; FERREIRA, A.S. Resistência parcial a diferentes raças de Colletotrichum graminicola. Relatório Técnico Anual do Centro Nacional de Pesquisa de Milho e Sorgo 1988-1991, Sete Lagoas, v. 4, p. 130 - 131, 1991.

CASELA, C.R.; FERREIRA, A.S.; SCHAFFERT, R. E. Sorghum diseases in Brasil. In: MILIANO, W.A.J.de; FREDERIKSEN, R.A.; BENGSTON, G.D. (Ed.). Sorghum and millets diseases: a second world review. Patancheru. ICRISAT, 1992.p.57-62.

GUIMARÃES, F.B.; CASELA, C.R.; SANTOS, F.G.; FERREIRA, A.S. Controle da antracnose do sorgo através da utilização de misturas de cultivares. Summa Phytopathologica, Jaboticabal, v. 24, n. 2, p. $131-135,1998$.

GUIMARÃES, F.B.; CASELA, C.R.; SANTOS, F.G.; FERREIRA, A.S. Avaliação da estabilidade fenotípica e previsibilidade da resistência de cultivares de sorgo a Colletotrichum graminicola. Summa Phytopathologica, Jaboticabal, v. 25, n. 1, p. 09 - 13, 1999.

HARRIS, H.B.; JOHNSON, B.J.; DOBSON, J.N.; LUTTRELL, E.S. Evaluation of anthracnose of grain sorghum. Crop Science, Madison, v. 4., p. 460 462, 1964.

LIMONARD, T. A modified blotter test for seed health. Netherlands Jounal of Plant Pathology, Wageningen, v. 72, p. 319 - 321, 1966.
PINTO, N. F. J. A.; FERNANDES, F. T. Avaliação de fungicidas no controle da mancha foliar do milho causada por Phyllosticta sp. (Phaeosphaeria maydis). Fitopatologia Brasileira, Brasília, v. 20, p. 333, 1995. Suplemento.

PINTO, N.F.J.A. Controle químico da ergot (Claviceps africana) ou doença açucarada do sorgo. In: CONGRESSO NACIONAL DE MILHO E SORGO, 22., 1998. Recife. Globalização e segurança alimentar: resumos. Recife: IPA, 1998. p. 169.

PINTO, N.F.J.A. Avaliação de fungicidas no controle de Sphacelia sorghi (Claviceps africana) agente etiológico da "ergot" ou doença açucarada do sorgo. Summa Phytopathologica, Jaboticabal, v. 25, n. 1, p. 4 - 8, 1999.

TARR, S.A.J. Diseases of sorghum, sudangrass, and broom corn. Kew: Commonwealth Mycologial Institute, $1962,380 \mathrm{p}$.

VALARINI, P.J.; LASCA, C.C.; VECHIATO, M.H.; SCHMIDT, J.R.; DION, P.; CHIBA, S. Tratamento de sementes de sorgo (Sorghum sp.) com fungicidas visando o controle de Colletotrichum graminicola (Ces.) Wils e outros fungos associados à sementes. Fitopatologia Brasileira, Brasília, v. 13, p. $238-243,1988$.

WARREN, H.P. Leaf anthracnose. In: FREDERIKSEN, R.A. (Ed.). Compedium of sorghum deseases. St. Paul: American Phytopathological Society, 1986.p.10-11. 